\title{
Enhancing image quality in cleared tissue with adaptive optics
}

Marc R. Reinig

Samuel W. Novak

Xiaodong Tao

Laurent A. Bentolila

Dustin G. Roberts

Allan MacKenzie-Graham

Sirie E. Godshalk

Mary A. Raven

David W. Knowles

Joel Kubby 


\title{
Enhancing image quality in cleared tissue with adaptive optics
}

\author{
Marc R. Reinig, ${ }^{\mathrm{a}, \star}$ Samuel W. Novak, ${ }^{\mathrm{a}}$ Xiaodong Tao, ${ }^{a}$ Laurent A. Bentolila, ${ }^{\mathrm{b}}$ Dustin G. Roberts, ${ }^{\mathrm{c}}$ \\ Allan MacKenzie-Graham, ${ }^{d}$ Sirie E. Godshalk, ${ }^{e}$ Mary A. Raven, ${ }^{f}$ David W. Knowles, ${ }^{g}$ and Joel Kubby ${ }^{a}$ \\ a University of California Santa Cruz, W.M. Keck Center for Adaptive Optical Microscopy, Baskin Engineering, 1154 High Street, \\ Santa Cruz, California 95064, United States \\ bUniversity of California, California Nanosystems Institute, Advanced Light Microscopy/Spectroscopy Laboratory, 570 Westwood Plaza, \\ Building 114, Los Angeles, California 90095, United States \\ 'UCLA Brain Mapping Center, 660 Charles E. Young Drive South, Los Angeles, California 90095, United States \\ ¿UCLA Neurology, 710 Westwood Plaza, PO Box 951769, 4256 Los Angeles, California 90095-1769, United States \\ eUniversity of California, Neuroscience Research Institute, Microscopy Facility, 3087 Calle Rosales, Santa Barbara, California 93105, \\ United States \\ 'UCSB's Office of Technology and Industry Alliances, 342 Lagoon Road, Mail Code 2055, Santa Barbara, California 93106-2055, United States \\ gLife Sciences Division, Berkeley Drosophila Transcription Network Project, Lawrence Berkeley National Laboratory, 1 Cyclotron Road, Berkeley, \\ California 94720, United States
}

\begin{abstract}
Our ability to see fine detail at depth in tissues is limited by scattering and other refractive characteristics of the tissue. For fixed tissue, we can limit scattering with a variety of clearing protocols. This allows us to see deeper but not necessarily clearer. Refractive aberrations caused by the bulk index of refraction of the tissue and its variations continue to limit our ability to see fine detail. Refractive aberrations are made up of spherical and other Zernike modes, which can be significant at depth. Spherical aberration that is common across the imaging field can be corrected using an objective correcting collar, although this can require manual intervention. Other aberrations may vary across the imaging field and can only be effectively corrected using adaptive optics. Adaptive optics can also correct other aberrations simultaneously with the spherical aberration, eliminating manual intervention and speeding imaging. We use an adaptive optics two-photon microscope to examine the impact of the spherical and higher order aberrations on imaging and contrast the effect of compensating only for spherical aberration against compensating for the first 22 Zernike aberrations in two tissue types. Increase in image intensity by $1.6 \times$ and reduction of root mean square error by $3 \times$ are demonstrated. $\odot 2016$ Society of Photo-Optical Instrumentation Engineers (SPIE) [DOI: 10.1117/1.JBO.21.12.121508]
\end{abstract}

Keywords: adaptive optics; CLARITY; multiphoton microscope; brain; neural circuits.

Paper 160432SSPR received Jun. 24, 2016; accepted for publication Sep. 19, 2016; published online Oct. 13, 2016.

\section{Introduction}

The intent of this research is to determine the relative effect of both spherical and nonspherical aberrations in various cleared tissues and to determine the benefit of compensating the nonspherical in addition to the spherical aberrations.

Our ability to look deep within tissue, using fluorescent imaging, is primarily limited by refractive index (RI) inhomogeneities and mismatches. ${ }^{1}$ These cause light scattering and distortion of the point-spread function (PSF) and result in reduced intensity, resolution, contrast, and penetration depth.

The light scattering qualities of tissue come primarily from high concentrations of lipids, proteins, cell structure, and inhomogeneities in RI. ${ }^{2-5}$ In brain tissue, the concentration of lipids is particularly strong.

The distortion of the PSF originates from several factors. The RI mismatch among the microscope lens, index matching fluid, coverslip, and the bulk RI of the tissue causes depth-dependent spherical aberration. The relative tilt of these optical elements, tissue structures, and the uneven distribution of the internal tissue structures relative to the lens can cause coma and astigmatism. Other inhomogeneities in the RI of the tissue cause higher order aberrations and further degradation. At shallow depths, below $100 \mu \mathrm{m}$, we can reduce aberrations by correcting for refractive aberrations using adaptive optics (AO).

However, beyond this relatively shallow depth $(\sim 100 \mu \mathrm{m})$, in brain and other tissue, for single photon imaging, scattering due to lipids or proteins becomes significant and, very soon after, no attempted improvement of the PSF can overcome the effects of the scattered light that reduces signal intensity, contrast, and resolution. ${ }^{3,6}$ Using fluorophores with longer excitation wavelengths allows us to penetrate deeper into the tissue, since scattering is inversely proportional to wavelength. ${ }^{7-10}$ Nevertheless, while using longer wavelength fluorophores or multiphoton excitation ${ }^{11}$ allows us to image even deeper, scattering still dominates within a few hundred $\mu \mathrm{m}$. This limits us currently to a penetration of $<1600 \mu \mathrm{m}$. $^{12}$

In order to image entire organs at high resolution one approach has been to serially section the tissue, image individual slices, and then recombine the images into a volume. This process is complicated by the physical damage from the slicing, and the fact that the deeper parts of each section still have reduced intensity, resolution, and contrast due to scattering and aberrations.

Fortunately, we can now treat entire samples to remove and replace the lipids with a variety of clarifying techniques. ${ }^{13,14}$ 
This yields samples with the scattering lipids removed, allowing us to image fluorophores deep into the now almost transparent structures, without the physical damage caused by sectioning. However, the PSF is still affected by multiple aberrations: spherical aberration due to the RI mismatches among the bulk RI of the cleared organ, index matching fluids, coverslip, and the lens becomes dominant at greater depths; coma and astigmatism due to the nonplanarity of those surfaces of the lens, coverslip, and tissue structures increase with depth; and, higher order aberrations due to the inhomogeneity of the RI of the tissue itself also increase with depth. ${ }^{15}$

A number of techniques have been used to correct for spherical aberrations. Many objective lenses have correction collars that can correct spherical aberration. However, they must be manually adjusted iteratively with the focus control to give the best correction. Some objective lenses have been designed to be matched to a specific clearing protocol. A more sophisticated approach to correct spherical aberration has been to synchronize a motorized correction collar with the stage height controller. Another approach is the use of AO to remove the spherical aberrations. ${ }^{16,17}$ AO systems can remove spherical aberration and the correct focal adjustment can be automatically applied without manual intervention. Unfortunately, after the spherical aberration is removed by any of the above means, the residual aberrations remain, including astigmatism, coma, and other aberrations, which will still degrade the PSF. These can only be effectively removed by an AO system.

In this paper, we characterize the impact of an AO system on clarified tissue imaging when used to remove aberrations beyond spherical aberration. To determine the effect of wavefront aberrations on the imaging of various clarified tissues, we compare images made of various tissues at various depths with and without AO correction, also focusing on the effect of spherical aberration compared to other aberrations. Due to the limit in the working distance of our objective, the maximum depth we could probe was $1500 \mu \mathrm{m}$. We have included representative images out of a large collection of images $(\sim 10)$ that clearly illustrate our points.

\section{Materials and Methods}

\subsection{Wavefront Metrics}

In order to provide an objective measure of a system's performance in imaging, we use the Strehl ratio. ${ }^{18-20}$ This is the ratio of a system's actual PSF to its theoretical diffraction limited PSF in the absence of aberrations. ${ }^{21}$ Aberrations cause light to be moved from the core of the PSF to other locations reducing both contrast and resolution. Systems with Strehl ratios of less than 0.3 can be considered poorly corrected, whereas systems with Strehl ratios above 0.8 are considered well-corrected. All aberrations contribute to the root mean square (RMS) wavefront error (measured in waves) and degrade the image quality; consequently, we also use the total RMS wavefront error $(\sigma)$ as a metric in our analysis.

The RMS wavefront error is calculated by reconstructing the wavefront measured using a Shack-Hartmann wavefront sensor. The wavefront is in turn decomposed to a set of 22 Zernike modes. These modes are orthogonal and normalized to a value of one wave of RMS error, so that, e.g., a mode value of 1.5 for any term would represent 1.5 waves of RMS error for that term. Total RMS wavefront error is calculated from the individual modes by, $\sigma=\left[\sum_{i}\left(\sigma_{i}\right)^{2}\right]^{1 / 2}$

The Strehl ratio $(\mathrm{Sr})$ is calculated from the RMS wavefront error $(\sigma)$ using the approximation ${ }^{21}$

$\mathrm{Sr}=\exp \left(-\sigma^{2}\right)$.

\subsubsection{Comparison of images}

When making comparisons among images, a slight focal shift ( $\sim 0$ to $3 \mu \mathrm{m}$ ) occurs due to spherical aberration. ${ }^{1}$ This is depth dependent. When removing spherical aberration during imaging this focal shift is removed causing the focus to be shifted to a slightly different plane. Consequently, when comparing the slices from stacks at the same ( $x$ and $y$ ) location, but differing in the amount of refractive aberrations removed, they are matched by features rather than measured depth or slice number. Depths shown are the nominal focal position as measured by the stage movement rather than the actual depth which takes into account the focal shift. For the images in this paper, the difference between corrected and uncorrected images was always less than $1 \%$.

\subsubsection{Reporting Zernike aberration values}

There are several different single-index methods for reporting Zernike aberrations (modes). We report them in Noll form as shown in Table $1 .^{22}$ Only the first 22 Zernike modes are reported in our calculations, since those of higher order do not contribute to a significant amount of RMS wavefront error. Zernike modes 1 to 4 (piston, tip, tilt, and focus) are ignored; piston being the absolute distance from the object, and tip, tilt, and focus being simply movements of the focal point in $X, Y$, and $Z$. Zernike modes are normalized so that a value of 1.0 for any mode corresponds to 1.0 wave of RMS error for that mode.

\subsection{Two-Photon Adaptive Optics Microscope}

A two-photon microscope ${ }^{23,24}$ was modified to include an AO system with an open-loop control system. Figure 1 shows the layout of the system. Two photon excitation was generated by

Table 1 First 11 Zernike aberrations in Noll single-index order.

\begin{tabular}{lc} 
Index & Zernike modes \\
\hline 1 & Piston \\
2 & Tip \\
3 & Tilt \\
4 & Defocus \\
5 & Oblique astigmatism \\
6 & Vertical astigmatism \\
7 & Vertical coma \\
8 & Horizontal coma \\
9 & Vertical trefoil \\
10 & Oblique trefoil \\
11 & Primary spherical \\
\hline
\end{tabular}




\section{Two photon optical system}

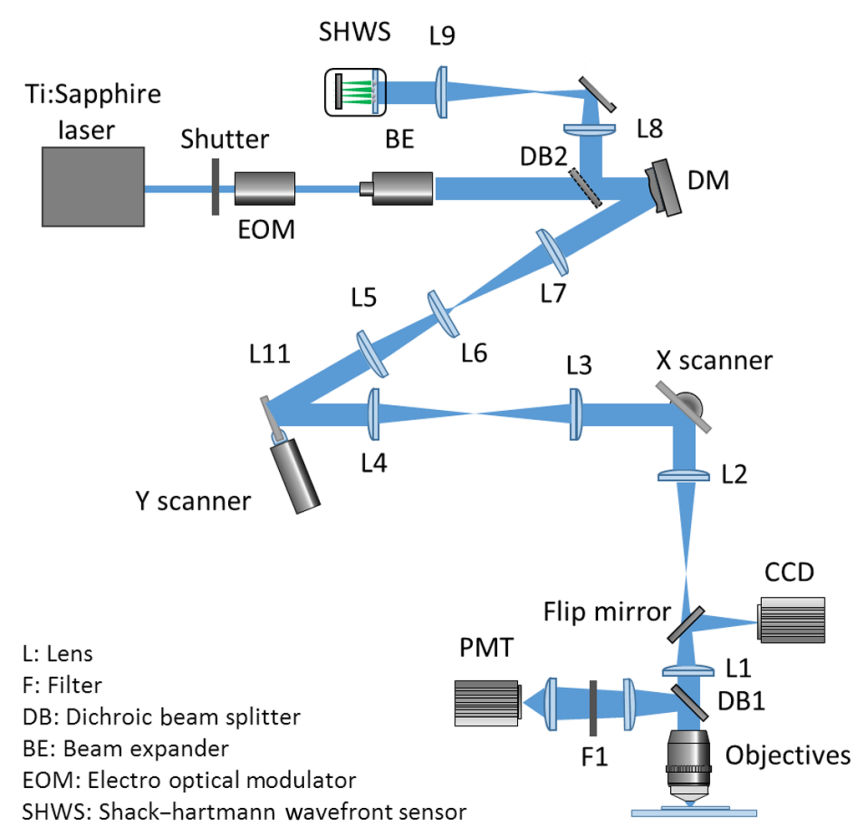

Fig. 1 Two-photon AO microscope setup.

a tunable (680 to $1080 \mathrm{~nm})$ mode locked Ti:Sapphire laser (140 fs, $80 \mathrm{MHz}$, Chameleon Ultra II, Coherent) the intensity of which was modulated by an electro-optic modulator (model 350- 80LA, Conoptics Inc.). A $25 \times$ water immersion objective with a numerical aperture of 1.05 was used (XLPlan N, Olympus Microscope, Center Valley, Pennsylvania) for imaging. The photomultiplier tube (H7422-20, Hamamatsu) was configured in a nondescanned mode, and collected the emitted light during imaging. To correct wavefront aberrations in the focused laser light, a deformable mirror (DM) (Boston Micromachines) with 140 actuators and $3.5 \mu \mathrm{m}$ of stroke was used. Wavefronts were measured with a $44 \times 44$-lenslet array in a ShackHartmann wavefront sensor (SHWS). The SHWS collected fully descanned light during wavefront measurement from selected points in the imaging plane. (For more complete details of the system, see Refs. 23 and 24). Imaging was done using a wavelength of $900 \mathrm{~nm}$ for two-photon excitation of the fluorophores. Wavefront measurements were made at a wavelength of $515 \mathrm{~nm}$. All measurements were made using an open-loop control method.

\subsection{CLARITY Mouse Brains}

Two different samples of CLARITY ${ }^{25}$ optically cleared mouse brains were used in this study.

The first was a whole optically cleared mouse brain labeled for the astrocytic marker glial fibrillary acidic protein (GFAP). The brain was placed on a slide and held stable with clay. Because the objective lens was a water immersion lens, water was placed on the surface as an index matching fluid to the lens. This led to some spherical aberration due to the mismatch of the water (RI 1.33) and the CLARITY brains (RI 1.454). ${ }^{26}$ No coverslip was used.

The second was a dissected section of optically cleared brain tissue from a Thy1-YFP mouse (Jackson Laboratories, Bar Harbor, Maine). Because of its thickness, the sample was enclosed in a custom slide chamber and immersed in FocusClear media (CelExplorer, Hsinchu, Taiwan). The chamber was then sealed with a coverslip to allow imaging, while preventing the loss of the media around the tissue during handling and transport. The difference between the index of the FocusClear (RI: $\sim 1.454$ ), the coverslip (Fisher Scientific \#2950-600621T RI: 1.517), and the water immersion fluid (RI: 1.33) caused some spherical aberration. This could be removed by either the collar on the lens or an AO system. However, the coma and astigmatigmatic aberrations introduced by the difference of planarity of the various tissue structures, the cover slip, and the lens could only be removed by an AO system. One can coalign the lens and the coverslip, but the sample has innate curvature that cannot be forced to be coplanar with them.

\subsection{2,2'-Thiodiethanol Mouse Spinal Cord}

A Thy1-YFP mouse spinal cord was clarified in a solution of $63 \%$ 2,2'-thiodiethanol (TDE) (RI $=\sim 1.45$ ), diluted with phosphate-buffered saline (PBS) ${ }^{14}$ and mounted in the same TDEPBS solution in a custom slide chamber (see Sec. 2.3).

\subsection{Analyzing the Measurements}

To determine the effect of wavefront aberrations on the imaging of the tissues, we compared images made at various depths with and without $\mathrm{AO}$ correction, and analyzed the contribution of spherical and nonspherical aberrations. Fluctuations with depth in the major Zernike aberrations were due to the fact that the wavefront was determined by using descanned light optically integrated during a scan. ${ }^{27}$ The descanned light came from each emitting fluorophore in the field of a given scan and thus the aberrations measured were an aggregate of the aberrations seen from each location. Since the location of the fluorophores was different for different scans, the aggregate of their aberrations was different and local apparent fluctuations occurred as we increased the depth. However, examination of the trend lines showed consistent overall increase with depth. We are experimenting on mitigating these fluctuations by measuring the wavefronts from a subset of elements located near the center of the scan.

\subsection{Measuring the Wavefronts}

\subsubsection{Reference wavefront measurements}

Images from the Shack-Hartmann (SHWF) sensor were used to determine an unaberrated reference wavefront. The reference images were taken from a 100-nm fluorescent bead on a slide under a \#1.5 (170- $\mu \mathrm{m})$ coverslip mounted with Vectashield (Vector Laboratories). This became the reference wavefront representing an unaberrated wavefront. The bead slide was then replaced with the sample slide for imaging and wavefront measurements were made at various depths.

\subsubsection{Aberration measurements}

The first 22 Zernike aberrations were measured, and the RMS wavefront error and Strehl ratio were determined. The excitation wavelength was centered at $900 \mathrm{~nm}$. The emission wavelength was centered at $515 \mathrm{~nm}$. Wavefronts were measured with descanned light at the emission wavelength. The correction during imaging was applied to the $900-\mathrm{nm}$ excitation after compensating for dispersion by manual optimization. Dispersion compensation was done in two ways. The first was to compensate for the dispersion of the optical system and attain the minimum pulse 

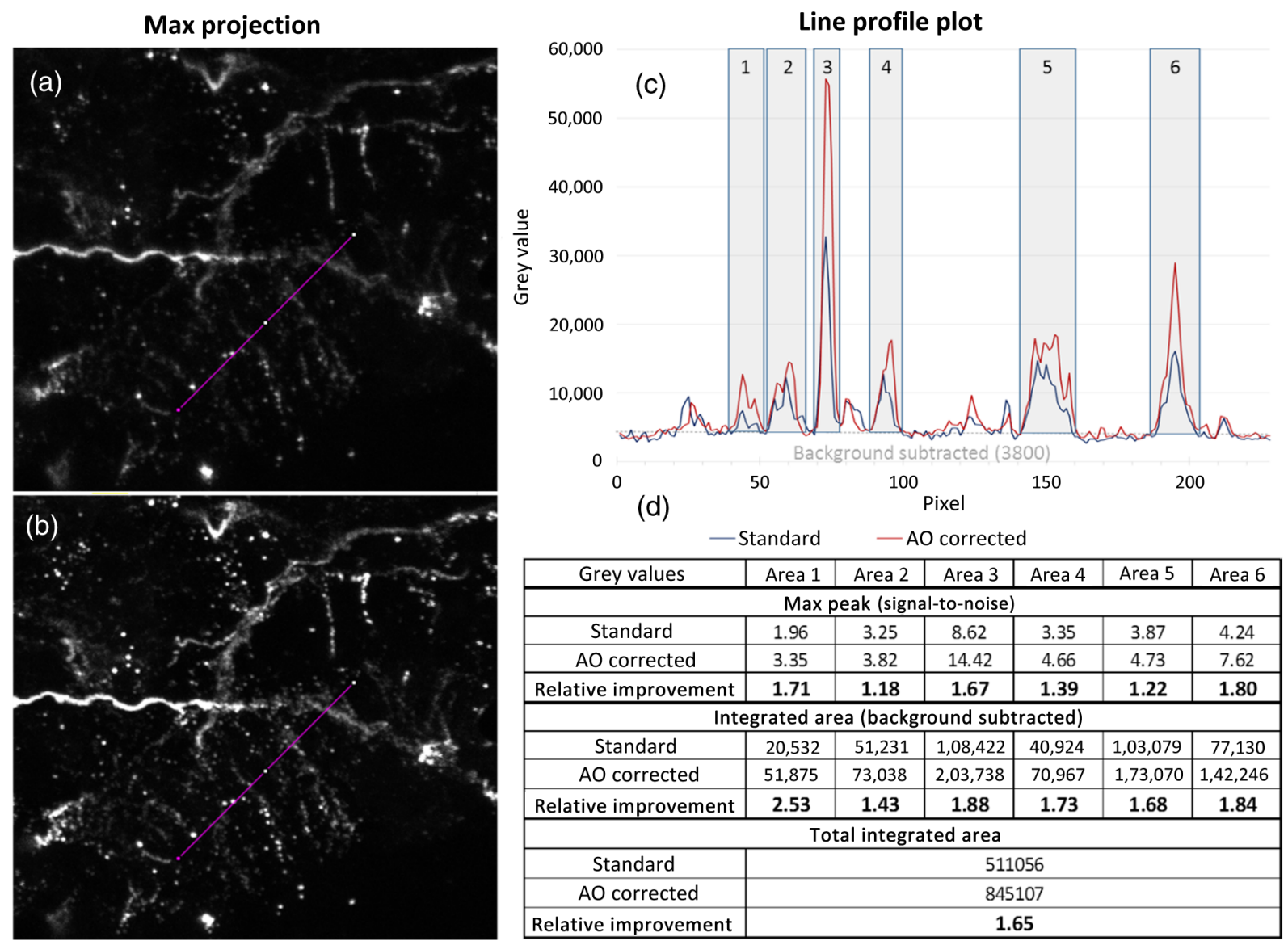

Fig. $212-\mu \mathrm{m}$ thick, two-photon image stack ( $Z$ step size $0.8 \mu \mathrm{m}$ ) of astrocytes at $1500-\mu \mathrm{m}$ depth in a CLARITY mouse brain. (a) Uncorrected maximal projection, (b) AO-corrected maximal projection, (c) line profile plot of the magenta lines in (a) and (b) with peaks highlighted as areas 1 to 6 , (d) data analysis of the line profile plot and areas 1 to 6 . Max peak values were obtained by dividing the peak value for each numbered area by the background, 3800. The background was an average of all values in the plot. The integrated area was obtained by adding all samples in the numbered area after subtracting the background. Total integrated area is the sum of all samples in the plot minus the background.

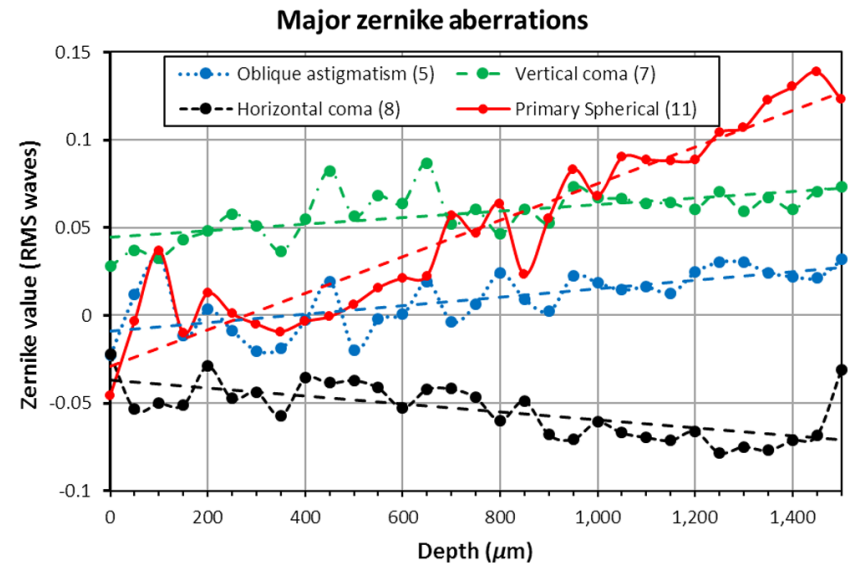

Fig. 3 Major Zernike aberrations and their trend lines. Aberrations increase as depth increases. NB: horizontal coma does not decrease with depth; it is increasing in a negative direction and adding RMS error to the wavefront. For clarity, only the largest magnitude aberrations are shown.

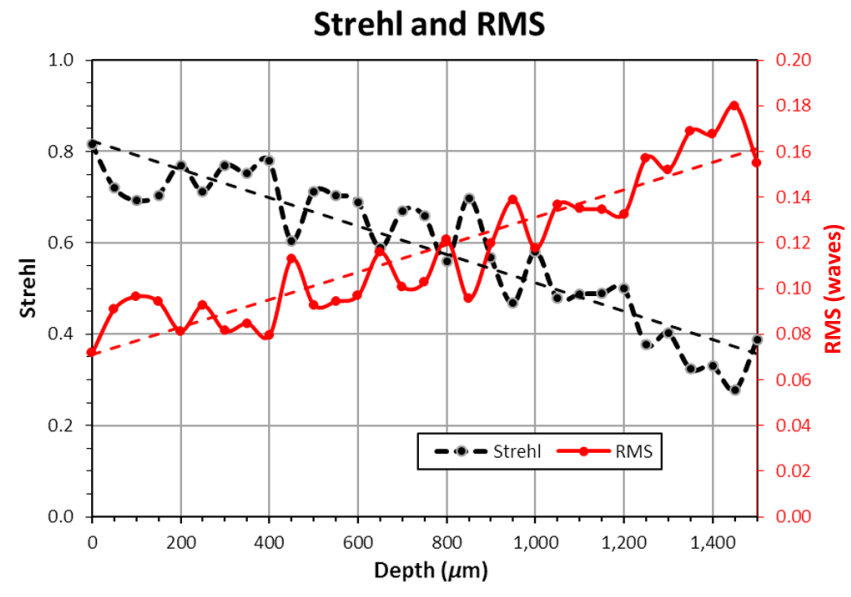

Fig. 4 Decreasing Strehl ratio and increasing RMS wavefront error versus depth in the optically cleared mouse brain indicated the diminished wavefront quality as focal depth increased. The RMS error at 0 depth is at the surface of the brain but imaged through a small amount of residual index matching fluid. The tilt of the organ relative to the objective and the irregularity of the surface add a slight amount of aberration. 
width of the laser. This was done using grating pairs in the actual laser system. The second was to compensate for the combined dispersion of the optical system and the tissue to compensate for the fact that the wave front measurement was made at the emission wavelength and the compensation was applied at the excitation wave length.

\section{Results}

\subsection{CLARITY Mouse Brains}

Aberrations in a CLARITY whole brain sample were measured at several different $(x, y)$ locations from the surface to a depth of $1500 \mu \mathrm{m}$ (limited by the working distance of our objective) in increments of $50 \mu \mathrm{m}$. At each increment, a $12-\mu \mathrm{m}$ stack was taken ( $Z$ step size $0.8 \mu \mathrm{m}$ ). For each stack of CLARITY

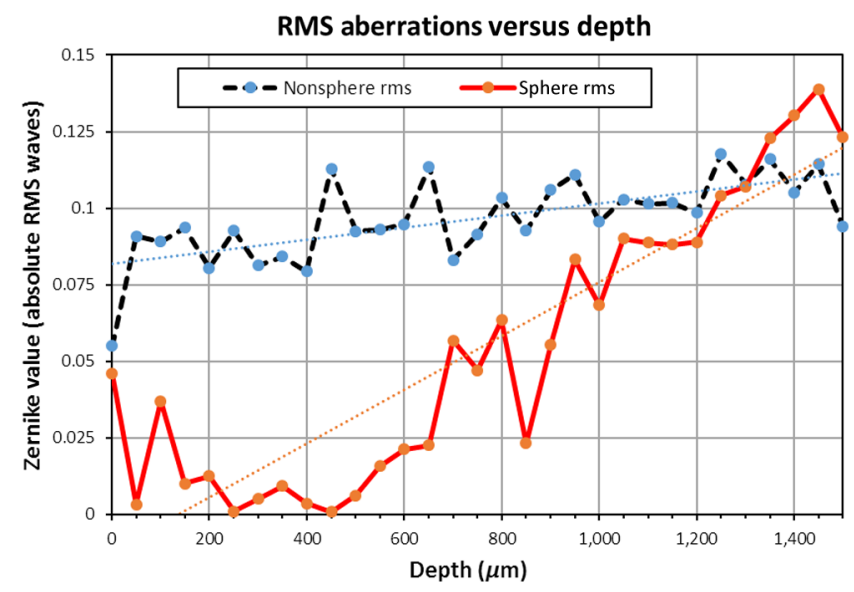

Fig. 5 Comparison of spherical versus other aberrations versus depth. See Sec. 2.5 for a discussion of the causes of variations of spherical aberrations with depth. micrographs, the first 22 Zernike aberrations were determined from a descanned section near the middle of the stack and used to calculate the corresponding voltages required to produce a complementary phase shift in the DM. Imaging of the brain sections was done at a depth of $500 \mu \mathrm{m}$.

We present two analyses here. The first is a summary of the aberrations in our deep scan to $1500 \mu \mathrm{m}$ in $50 \mu \mathrm{m}$ steps. Here we focused on the comparative amount of spherical aberration to other aberrations. Spherical aberrations can be removed by a collar on the objective or by an AO system, whereas the other nonspherical aberrations can only be removed by an AO system.

The second analysis was of the effect on imaging of removing only spherical aberrations compared to removing all aberrations. This was performed at a depth of $500 \mu \mathrm{m}$. We measured the wavefront and calculated RMS wavefront error and Strehl ratio in a variety of locations on the sample. Imaging was done without $\mathrm{AO}$, with only spherical aberration compensated by $\mathrm{AO}$, and with all aberrations compensated by $\mathrm{AO}$.

\subsubsection{Comparing aberrations from 0 to $1500 \mu \mathrm{m}$}

We examined a whole CLARITY treated mouse brain immunostained for the astrocytic marker (GFAP). At 1500- $\mu$ m depth, we measured the wavefront and calculated RMS wavefront error and the Strehl ratio (Fig. 4). As expected, we found a decreased Strehl ratio and increased RMS wavefront error values as the depth of the measurements increased.

Imaging with $\mathrm{AO}$ wavefront correction resulted in an increase of the signal-to-noise ratio in the background corrected measurements from $43 \%$ to $253 \%$ (Fig. 2). AO correction revealed fine details of small astrocytic processes. Astrocytes are involved in regulating neural signaling and tissue homeostasis and are important signal mediators between the brain and the vasculature. Most of these processes are performed through contact with astrocytic end feet, a process that could be studied in greater detail with the help of AO.
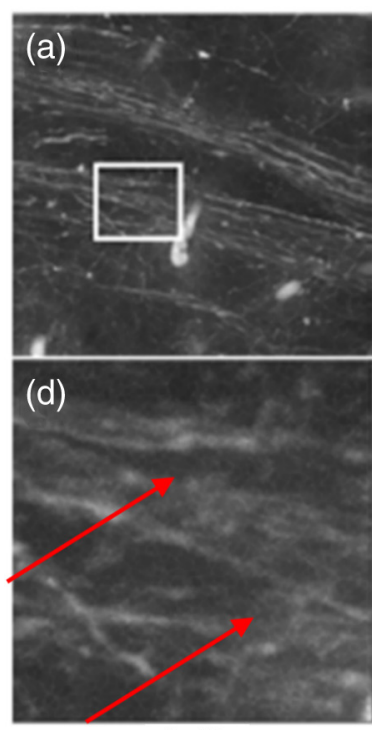

No AO
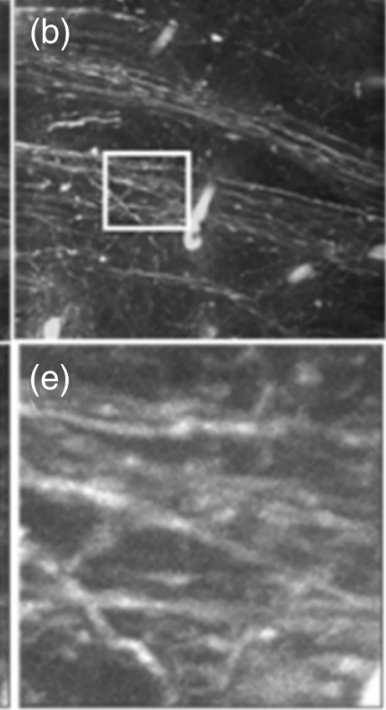

Sphere removed
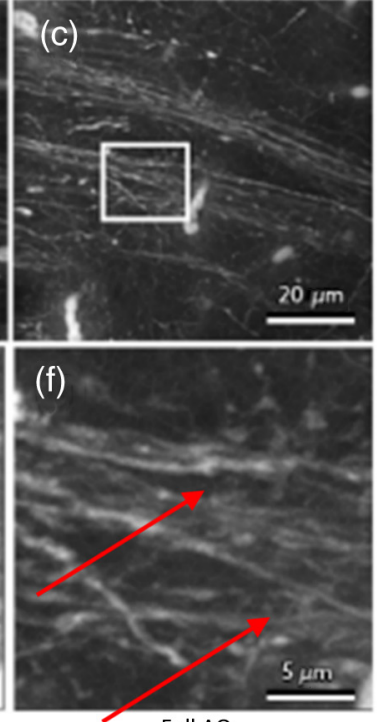

Full AO

Fig. 6 Brain slice, neurite bundle at a depth of $500 \mu \mathrm{m}$. (a)-(c) Field of view is $85 \mu \mathrm{m} \times 85 \mu \mathrm{m}$ and is a maximal $Z$ projection of 19 sections spaced at $1.0-\mu \mathrm{m}$ intervals. (a) No aberration correction. (b) Only spherical aberration corrected. (c) First 22 Zernike aberrations corrected. The insets are shown in (d)-(e). (e) Removing spherical aberration only increased resolution somewhat. (f) Removing the first 22 Zernike aberrations showed a significant improvement. 
Figure 3 shows the increasing contribution of the major nonspherical aberrations compared to the spherical aberration. Figure 4 shows the reduction in the Strehl ratio compared to the total RMS wavefront error as the depth increased. At a depth of $1500 \mu \mathrm{m}$, the RMS wavefront error contributed by the spherical aberration was approximately equal to that of the nonspherical aberrations, as shown in Fig. 5. See Sec. 2.5 for an analysis of the fluctuations of the aberrations with depth.

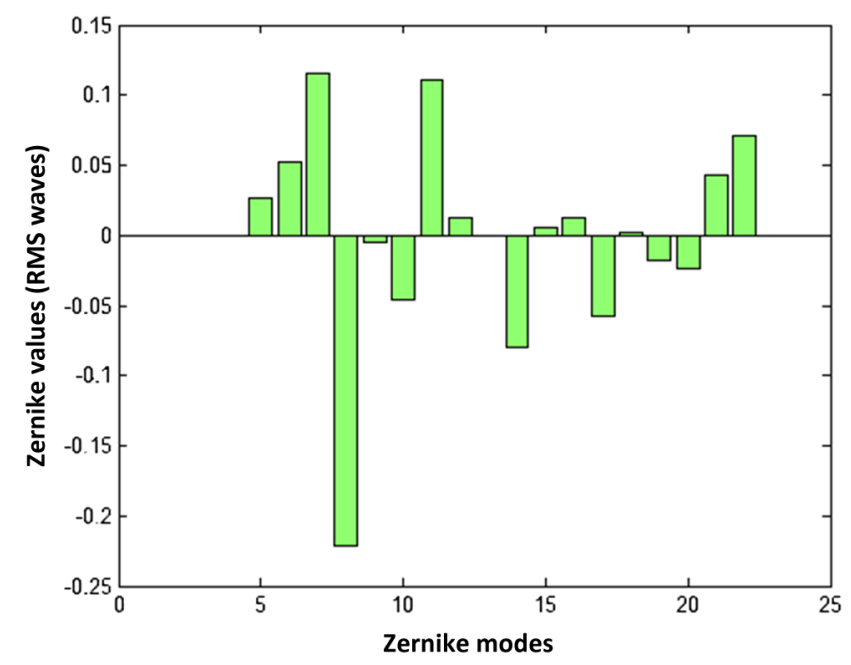

Fig. 7 Brain slice at $500 \mu \mathrm{m}$. Zernike modes (waves RMS) (in Noll order) of the neurite bundle wavefront prior to AO correction (piston, tip/tilt, focus removed) (spherical aberration is index 11, at $\sim 0.1$ ). The large value of index 8 (horizontal coma at $\sim 0.22$ ) was due to the combination of the angle of the coverslip and tissue upper surface relative to the objective lens.
In Fig. 3 it is important to note that horizontal coma was not decreasing as depth increased, it was increasing in a negative direction and thus adding RMS wavefront error to the total RMS of the wavefront. In Fig. 4, the RMS error at 0 depth is at the surface of the brain, but is imaged through a small amount of residual index matching fluid. The tilt of the organ relative to the objective and the irregularity of the surface add a slight amount of aberration over the field.

Figure 5 shows that the nonspherical aberrations were significant, increasing, and dominated until a depth of almost $1500 \mu \mathrm{m}$. Spherical aberration is stronger than the nonspherical and increases faster with depth. However, all aberrations do increase with depth and all contribute to a reduction in imaging quality. Only an AO system can effectively reduce these aberrations. At larger depths, spherical aberrations would dominate. However, the amount of nonspherical aberrations at these depths would degrade imaging even if all spherical aberrations were removed.

\subsubsection{Analysis of aberrations in CLARITY mouse brains sections at $500-\mu \mathrm{m}$ depth}

The relationships among the different aberrating components were determined by analyzing images and corresponding wavefront measurements under three conditions: (1) without $\mathrm{AO}$, (2) with only spherical aberration compensated by AO, and (3) with all aberrations corrected with AO.

Stacks were collected at a depth of $\sim 500 \mu \mathrm{m}$ below the coverslip, where features were readily observable without AO but were obviously improved with the appropriate AO correction. Figure 6 is a representative images showing the improved resolution upon correction for spherical aberration and the even greater correction achieved when all Zernike modes were (a)

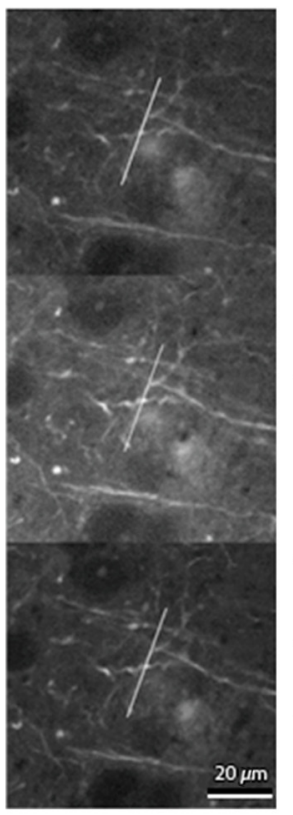

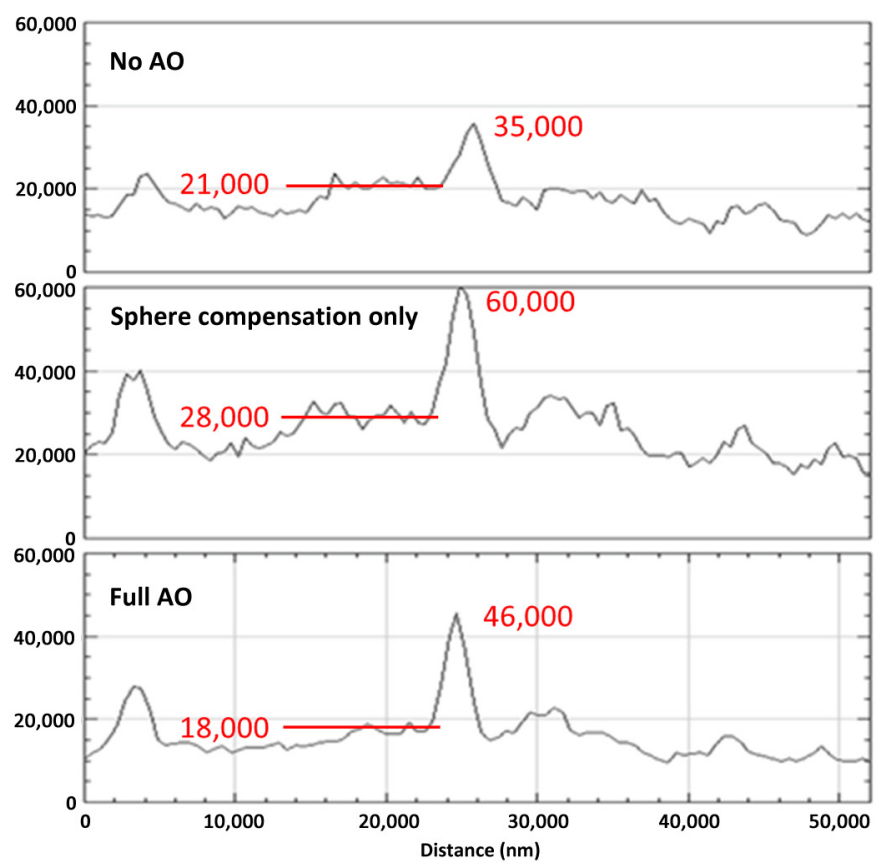

Fig. 8 Images of a CLARITY brain slice neurite bundle at a depth of $500 \mu \mathrm{m}$. (a) No AO correction: $S / N=1.7$. (b) Correction for spherical aberration only showed an $S / N=2.1$ an increase of $29 \%$. (c) First 22 Zernike aberrations corrected showed an $S / N=2.6$ an increase of $19 \%$ over correcting for spherical aberration only and a total increase of $50 \%$. The reduction in intensity in (c) is because it was measured last and some photobleaching had occurred. Scale bar is $20 \mu \mathrm{m}$. The horizontal bars indicate the noise floor used in calculations of the $S / N$. 


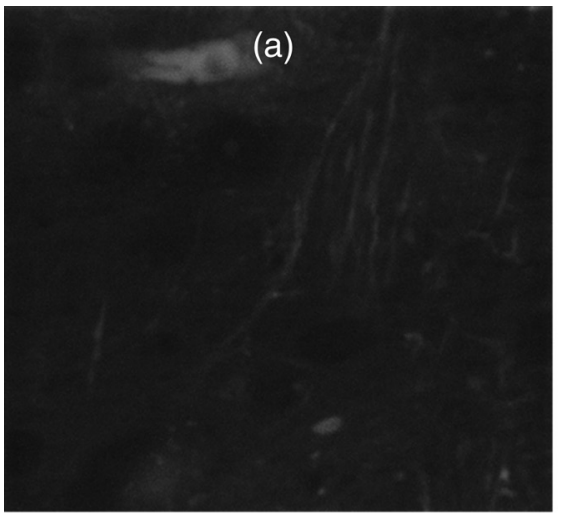

No AO

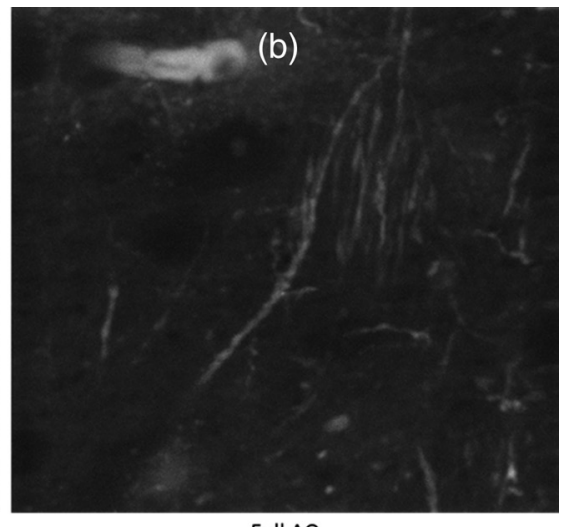

Full AO

Fig. 9 Brain slice at a depth of $500 \mu \mathrm{m}$. A dendritic segment with dendritic spines is centered in the field of view. The fine structures were more readily resolved after AO correction. Field of view is $55 \mu \mathrm{m} \times 55 \mu \mathrm{m}$, maximal $Z$-projection of 20 sections at $500-\mathrm{nm}$ intervals.

considered for the wavefront correction. Comparing Figs. 6(d) and 6(f), it shows that the signal-to-noise ratio and the resolution in (f) are visibly greater than (d). This can be seen as illustrated by the arrows. Figure 7 clearly shows that the amount of cumulative RMS wavefront error generated by nonspherical aberrations was significant (spherical aberration is index 11 and has a value approximately of 0.1 ). The large value of index 8 (horizontal coma at $\sim 0.22$ ) was due to the combination of the angle of the coverslip and the upper surface of the tissue relative to the objective lens and could only be effectively removed with an AO system. The relative tilt of various tissue layers with differing bulk RI with respect to each other, the cover slip, or objective will have the same effect and cannot be corrected by aligning to a particular layer since they are not colinear. ${ }^{28}$

Figure 8 shows a CLARITY brain slice where contrast was significantly improved over correction for spherical aberration only. With full AO correction, the PSF had a smaller axial profile as well as a smaller lateral profile. This reduced axial profile reduced imaging of structures that are above or below the level of the current slice. Removing spherical aberration only increased the $S / N 29 \%$. Removing all of the first 22 Zernike aberrations increased the $S / N$ a total of $50 \%$. Photobleaching

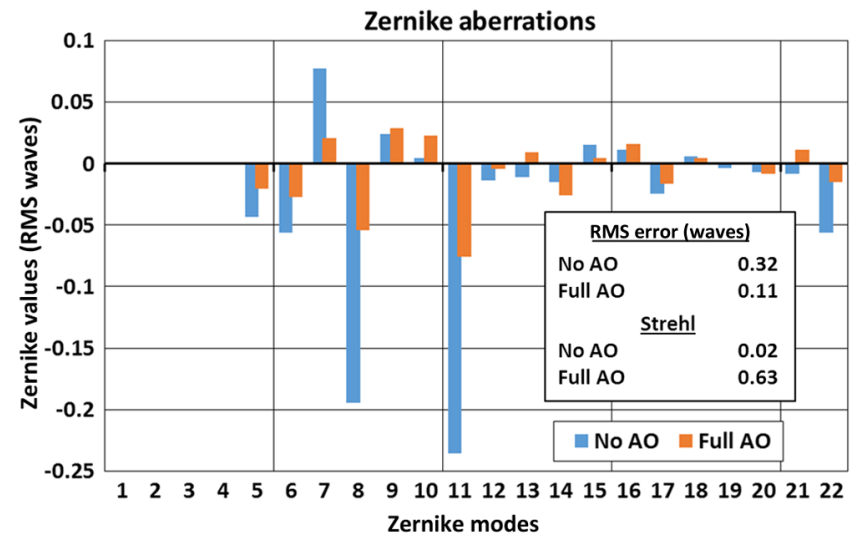

Fig. 10 Zernike modes of the wavefront aberrations for the images in Fig. 9 illustrating the improved quality of the wavefront after AO correction. Shown before and after AO compensation. (Spherical aberration is index 11, at approximately -0.23 .) The large value of index 8 (horizontal coma at $\sim 0.19$ ) was due to the combination of the angle of the coverslip and tissue upper surface relative to the objective lens. from multiple scans decreased the overall intensity in the full AO image since it was taken last.

Figures 9 and 10 show images and wavefront measurements across additional samples. Figure 9 shows images at a depth of $500 \mu \mathrm{m}$, both with and without $\mathrm{AO}$ compensation. It can be seen in Fig. 10 that even with AO compensation there were still significant residual aberrations. These prevented the images with AO correction from achieving maximum enhancement. This shortcoming in our compensation was due to a combination of the size of the aberrations compared to the capabilities of the DM and a limitation in our open-loop control system where we cannot sense or compensate adequately in the presence of large aberrations. We are addressing this by optimizing our open-loop algorithm to correctly sense a larger range of aberrations, incorporating an optional closed-loop mode, which has proven capable of reducing residual errors to a minimum, and measuring and compensating for the effects of dispersion. All of the residual Zernike values were below 0.1, most were below 0.05. and RMS wavefront error was reduced by a factor of 3 (from 0.32 to 0.11 ). Additionally, we were able to significantly increase the Strehl ratio from 0.02 (poorly corrected) to 0.63 .

\subsection{Clarified Mouse Spinal Cord}

This neurite fascicle from the Thy1-YFP mouse spinal cord was imaged in $1 \mu \mathrm{m}$ steps to a total depth of $188 \mu \mathrm{m}$ to show the impact of AO correction for different structures (e.g., the spinal cord instead of dendrites and spines). Aberrations were measured at each step. Data shown were at a depth of $133 \mu \mathrm{m}$.

Figure 11 shows mouse spinal cord at a depth of $133 \mu \mathrm{m}$. The analysis shows that removing the first 22 Zernike aberrations increased the signal level by a factor of 1.6, demonstrating the improvement in the signal and potential signal-to-noise ratio that is possible in clarified samples using AO even at this superficial depth.

Figure 12 shows measurements from a depth of $133 \mu \mathrm{m}$. Spherical aberration and vertical coma clearly dominated the aberrations, each contributing approximately half a wave of RMS error. This resulted in a combined RMS error of 0.74 waves. Removing only the spherical aberration would still have left a significant RMS error of approximately half a wave RMS. 

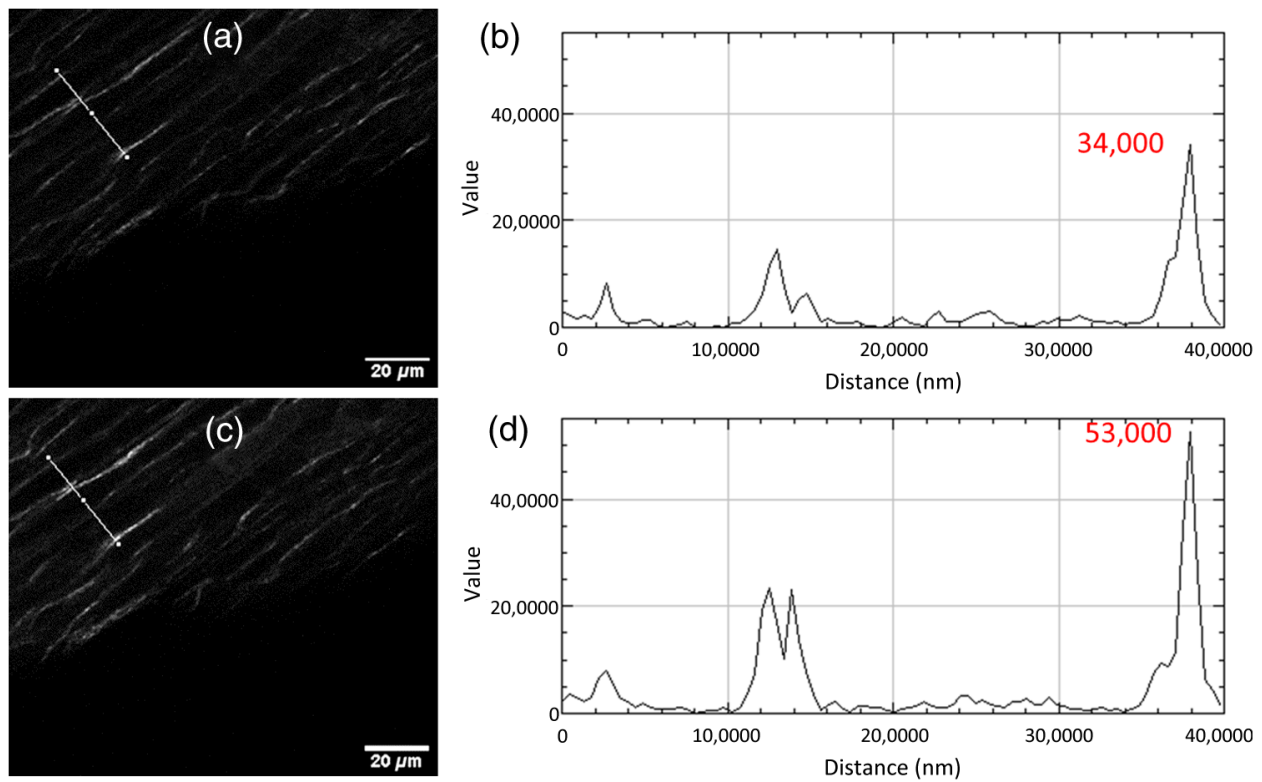

Fig. 11 Mouse spinal cord at a depth of $133 \mu \mathrm{m}$. (a) No AO applied, both intensity and contrast are low. (b) Line plot of the pixel values in (a). (c) Applying AO for the first 22 Zernike modes, increased the signal, allowing weak signals to be better imaged. (d) Line plot of the pixel values in (c). Signal level was increased by a factor of 1.6. Scale bar is $20 \mu \mathrm{m}$. Contrast in this image has been increased for publication.

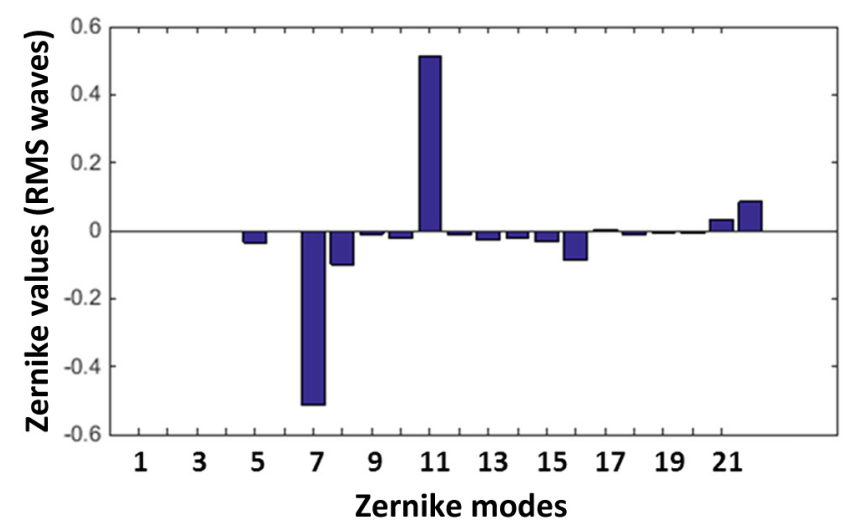

Fig. 12 Aberrations in a mouse spinal cord measured at a depth of $133 \mu \mathrm{m}$.

\section{Conclusions}

Clearing tissue allows examination of deep structures without the degrading effects of scattering found in uncleared tissues. However, refractive aberrations still degrade the quality of the image as we peer deeper into the structure. The microscope objective collar can remove the spherical aberration, which increases with depth, but other accumulated aberrations also increase with depth and cannot be removed without a higherorder AO system. We have analyzed various tissues, measured the increase in aberrations with depth, and calculated the impact on the Strehl ratio and RMS wavefront error, as well as image quality, at various depths.

For CLARITY mouse brains, we were able to reduce the RMS wavefront error by a factor of three, from 0.32 to 0.11 , even at a superficial depth of $500 \mu \mathrm{m}$. We were able to significantly increase the Strehl ratio from 0.02 (poorly corrected) to 0.63 at the same depth. As we probe deeper into CLARITY brains, refractive aberrations other than spherical aberration will only increase, requiring the use of $\mathrm{AO}$ to allow us to image structures with high Strehl and signal-to-noise ratio.

For the TDE-cleared mouse spinal cord, the intensity of the image was increased by a factor of 1.6. Nonspherical aberration contributed over 0.5 wave of RMS error. This is a significant amount of aberration if only the spherical aberration was removed.

Both spherical and nonspherical aberrations increase with depth. Beyond $1500 \mu \mathrm{m}$, spherical aberrations would dominate nonspherical aberrations. However, the amount of nonspherical aberrations would still be sufficient to significantly degrade imaging even if all spherical aberrations were removed by using protocol-specific objectives and/or adjusting the objective's collar. These residual aberrations must be removed using an AO system.

\section{Acknowledgments}

The results presented herein were obtained at the W.M. Keck Center for Adaptive Optical Microscopy (CfAOM) at the University of California, Santa Cruz. The CfAOM was made possible by the generous financial support of the W.M. Keck Foundation. This material is based upon work supported by the UC Office of the President for the UC Work Group for Adaptive Optics in Biological Imaging, by the Multicampus Research Programs and Initiatives, Grant No. MR-15-327968. This material is also based upon work supported by the National Science Foundation under Grant Nos. 1353461 and 1429810. Any opinions, findings, and conclusions or recommendations expressed in this material are those of the authors and do not necessarily reflect the views of the National Science Foundation.

\section{References}

1. S. Hell et al., "Aberrations in confocal fluorescence microscopy induced by mismatches in refractive index," J. Microsc. 169(3), 391-405 (1993). 
2. P. F. Mullney and P. N. Dean, "Cell sizing: a small-angle light-scattering method for sizing particles of low relative refractive index," Appl. Opt. 8(11), 2361 (1969).

3. R. A. Meyer, "Light scattering from biological cells: dependence of backscatter radiation on membrane thickness and refractive index," Appl. Opt. 18(5), 585 (1979).

4. R. A. Meyer and A. Brunsting, "Light scattering from nucleated biological cells," Biophys. J. 15(3), 191-203 (1975).

5. B. Türke et al., "Fourier optical approach to the extraction of morphological parameters from the diffraction pattern of biological cells," Appl. Opt. 17(17), 2754 (1978).

6. S. Johnsen and E. A. Widder, "The physical basis of transparency in biological tissue: ultrastructure and the minimization of light scattering," J. Theor. Biol. 199(2), 181-198 (1999).

7. G. Mie, "Beiträge zur optik trüber medien, speziell kolloidaler metallösungen," Ann. Phys. 330(3), 377-445 (1908).

8. J. W. Strutt, "LVIII. On the scattering of light by small particles," Philos. Mag. J. Sci. 41(275), 447-454 (1841).

9. N. Ji, T. R. Sato, and E. Betzig, "Characterization and adaptive optical correction of aberrations during in vivo imaging in the mouse cortex," Proc. Natl. Acad. Sci. U. S. A. 109(1), 22-27 (2012).

10. K. Wang et al., "Direct wavefront sensing for high-resolution in vivo imaging in scattering tissue," Nat. Commun. 6, 7276 (2015).

11. W. Denk, J. H. Strickler, and W. W. Webb, "Two-photon laser scanning fluorescence microscopy," Science 248(4951), 73-76 (1990).

12. L.-C. Cheng et al., "Measurements of multiphoton action cross sections for multiphoton microscopy," Biomed. Opt. Express 5(10), 3427-3433 (2014).

13. K. Chung et al., "Structural and molecular interrogation of intact biological systems," Nature 497(7449), 332-337 (2013).

14. I. Costantini et al., "A versatile clearing agent for multi-modal brain imaging," Sci. Rep. 5, 9808 (2015).

15. V. Marx, "Microscopy: seeing through tissue," Nat. Methods 11(12), 1209-1214 (2014).

16. P. Kner et al., "High-resolution wide-field microscopy with adaptive optics for spherical aberration correction and motionless focusing," J. Microsc. 237(2), 136-147 (2010).

17. P. S. Tsai et al., "Spherical aberration correction in nonlinear microscopy and optical ablation using a transparent deformable membrane," Appl. Phys. Lett. 91(19), 191102 (2007).

18. K. Strehl, "Aplanatische und fehlerhafte abbildung im fernrohr," Z. Instrumentenkd. 15, 362-370 (1895).

19. K. Strehl, "Über luftschlieren und zonenfehler," Z. Instrumentenkd. 22, 213-217 (1902)

20. V. N. Mahajan, "Strehl ratio for primary aberrations: some analytical results for circular and annular pupils," J. Opt. Soc. Am. 72(9), 1258 (1982).

21. J. W. Hardy, Adaptive Optics for Astronomical Telescopes, Oxford University Press, New York, New York (1998).
22. R. J. Noll, "Zernike polynomials and atmospheric turbulence," J. Opt. Soc. Am. 66(3), 207 (1976).

23. X. Tao et al., "Adaptive optics two photon microscopy with direct wavefront sensing using autofluorescent guide-stars," Proc. SPIE 8978, 89780D (2014).

24. X. Tao et al., "Adaptive optical two-photon microscopy using autofluorescent guide stars," Opt. Lett. 38(23), 5075 (2013).

25. K. Chung and K. Deisseroth, "CLARITY for mapping the nervous system," Nat. Methods 10(6), 508-513 (2013).

26. R. Tomer et al., "Advanced CLARITY for rapid and high-resolution imaging of intact tissues," Nat. Protoc. 9(7), 1682-1697 (2014).

27. K. Wang et al., "Rapid adaptive optical recovery of optimal resolution over large volumes," Nat. Methods 11(6), 625-628 (2014).

28. E. Ibrahimovic et al., "Deep tissue wavefront estimation for sensorless aberration correction," in MATEC Web of Conf., Vol. 32, p. 7001 (2015).

Marc R. Reinig received his BS degree in engineering from San Jose State University in 1975. He is a PhD graduate student at the University of California Santa Cruz at the W.M. Keck Center for Adaptive Optical Microscopy. He is the author of three journal papers. He has worked extensively in industry and retired from the University of California Lick Observatory. His current research interests are adaptive optics for microscopy, biological tomography, and superresolution imaging.

Samuel W. Novak received his BS and MS degrees in biomedical toxicology and neuroscience from the University of Guelph and University of Victoria in 2011 and 2015, respectively. He is a specialist at the W.M. Keck Center for Adaptive Optical Microscopy at the University of California Santa Cruz. His research interests include applications of adaptive optics in biological imaging, characterizing optical properties of biological tissues, high resolution imaging of large volumes of tissues, and correlative light/electron microscopy.

Xiaodong Tao received his $\mathrm{PhD}$ in mechanical engineering from Korea Advanced Institute of Science and Technology. He is an assistant project scientist of electrical engineering at the University of California, Santa Cruz. His research is in the area of adaptive optics and microscopy with applications in biological imaging.

Joel Kubby is in the Baskin School of Engineering at UC Santa Cruz. His research is in the area of biological imaging using adaptive optics. Prior to joining UC Santa Cruz in 2005, he was a member of technical staff at the Xerox Research Center in Rochester, New York, from 1987 to 2005, and at the Bell Telephone Laboratories in Murray Hill, New Jersey, from 1985 to 1987, working in the area of scanning tunneling microscopy (STM).

Biographies for the other authors are not available. 